\title{
Cervical isometric strength and range of motion of elite rugby union players: a cohort study
}

\author{
David F Hamilton ${ }^{1 *}$ and Don Gatherer ${ }^{2}$
}

\begin{abstract}
Background: Head and neck injury is relatively common in Rugby Union. Despite this, strength and range-of-motion characteristics of the cervical spine are poorly characterised. The aim of this study was to provide data on the strength and range-of-motion of the cervical spine of professional rugby players to guide clinical rehabilitation.

Methods: A cohort study was performed evaluating 27 players from a single UK professional rugby club. Cervical isometric strength and range-of-motion were assessed in 3 planes of reference. Anthropometric data was collected and multivariate regression modelling performed with a view to predicting cervical isometric strength.

Results: Largest forces were generated in extension, with broadly equal isometric side flexion forces at around $90 \%$ of extension values. The forwards generated significantly more force than the backline in all parameters bar flexion. The forwards had substantially reduced cervical range-of-motion and larger body mass, with differences observed in height, weight, neck circumference and chest circumference $(p<0.002)$. Neck circumference was the sole predictor of isometric extension (adjusted $R^{2}=30.34$ ).
\end{abstract}

Conclusion: Rehabilitative training programs aim to restore individuals to pre-injury status. This work provides reference ranges for the strength and range of motion of the cervical spine of current elite level rugby players.

Keywords: Rugby, Cervical spine, Strength, Range of motion

\section{Background}

Rugby union's move to professionalism over recent years has markedly altered the physiques of elite level players; Dramatic increases in body mass, strength and power [1-3] has resulted in larger magnitude impact forces in the contact phases of the game. This has perhaps been driven by the strong association seen between teams containing the largest players and success in the professional game [1]. Alongside this change in player morphology, the number of contact events occurring during a match has risen four-fold [4]. Perhaps unsurprisingly, rugby union carries a comparatively high risk injury when compared with other sports $[5,6]$.

Tackle based sports such as rugby exposes the cervical spine to potentially injurious forces, which are moderated by the musculoskeletal tissues [7]. While the relatively few catastrophic cervical injuries garner the most research and media interest, it has been suggested that

\footnotetext{
* Correspondence: d.f.hamilton@ed.ac.uk

${ }^{1}$ Department of Trauma and Orthopaedics, University of Edinburgh, Chancellor's Building, 49 Little France Crescent, Edinburgh EH164SB, UK Full list of author information is available at the end of the article
}

the cumulative effects of these continuous shear and compression forces can have great impact on range of motion [8], muscle function [9] and proprioception [8,9] of the cervical spine. This may impair the spinal reflexes that act to stabilise and protect this vulnerable region, perhaps predisposing to further injury.

Head and neck injuries account for around $30 \%$ of all rugby injury events $[6,10,11]$. Considering that such injuries may involve multiple scans and clinical assessments, a substantial proportion of medical team input is devoted to treating and rehabilitating players to preinjury status. It is surprising then that 'pre-injury' strength and range of motion characteristics of the cervical spine in professional rugby players has gone largely unreported in the literature. Conditioning of the cervical musculature has been proposed as a sensible (though unproven) method of mitigating neck injury $[12,13]$, while post-injury muscle strength training is also a routinely employed rehabilitative tool, and is a primary treatment modality for neck pain [14]. Appropriate therapy intervention and muscle training loads cannot be applied without reference 
to expected maximal loading, with inadequate training loads clearly resulting in incomplete rehabilitation.

The primary aim of this study was to provide data on typical isometric strength parameters and range of motion of the cervical spine of current professional rugby players to guide clinical rehabilitation. Our secondary aim was to model factors predictive of cervical strength to aid clinical interpretation of recovery in the absence of individual specific muscle strength data.

\section{Methods}

\section{Study design and population}

This study was a retrospective evaluation of existing performance data. Assessment of cervical range of motion and isometric muscle strength was performed at a Welsh professional rugby union club on a single occasion (in 2011). Testing was carried out by the same individual. All players contracted to the club were invited to participate, providing they were free from head, neck or upper body injury. Three players were excluded with injury; five were not present at the testing session. No available players declined participation; 27 players consented to testing. Ethical approval was sought for this study form the South East Scotland REC; however the opinion was that this was retrospective evaluation of blinded data and did not require specific approval as the players originally consented to testing and the club consented to release blinded data for the purpose of evaluation.

\section{Assessments}

Anthropometric measures were made of player height (Leicester Height Measure; SECA, UK), weight (medical grade mechanical flat scales; SECA, UK), neck circumference and chest circumference (standard fabric tape measure). Neck girth was assessed with the head in the anatomical position, using the thyroid cartilage as a reference position. Care was taken to tightly fasten the fabric measure around the neck but avoiding compression of the underlying tissues. Chest girth was assessed at full expiration, measuring the upper chest on the level of junction between the deltopectoral groove and tip of anterior axillary fold.

Cervical range of motion was assessed in three planes of reference (sagittal, frontal and transverse) using the Cervical Range of Motion Instrument (Performance Attainment Associates, Minnesota, USA) which has been thoroughly validated in various populations [15-18]. The assessment followed the test protocol advocated by Lark and McCarthy (2007), which has been previously described in detail [8]. Briefly, the participant was seated on a static chair with adjustable height so that the hips, knee and feet were positioned at $90^{\circ}$, and the head placed in the neutral anatomic position. An initial warm-up and familiarisation session practicing all movements to be assessed (flexion, extension, lateral flexion and rotation) was performed. Upon instruction, the participants moved their heads in the test parameter (looking directly ahead) and held the end of range position to allow the assessor to record the angle achieved, following which, the individual returned their head to the neutral position. Three separate measures were made in each direction, the mean of which is reported.

\section{Neck strength assessment}

Isometric cervical muscle testing is well validated [19-21]. We assessed maximal voluntary isometric cervical muscle strength in three planes of reference with the GS Gatherer and GS Analysis Suite (Gatherer Systems Ltd, Aylesbury); a custom-built device based on a $300 \mathrm{Kg}$ load cell and bespoke software system. The test was performed employing a previously reported protocol [22], specifically validated in young rugby players [13], subjecting the neck to manually controlled linear incremental loading to test positional failure in the absence of pain or neurological symptoms (which stopped the test). The head was held in the neutral anatomic position at all times throughout the test. Peak isometric force was logged at the point of head movement with loss of test position. Loading was applied and data were recorded at $20 \mathrm{~Hz}$. Peak isometric force generated by the musculature was defined as the maximal load recorded during the test procedure. An average of 3 tests is reported.

\section{Statistical analysis}

Data were assessed for normality and are reported as means with standard deviations as a measure of dispersion. Independent samples t-tests were used to assess differences in continuous variables between groups unless otherwise stated. Significance was accepted as $\mathrm{p}<$ 0.05 incorporating the Benjami-Hochberg FDR correction $[23,24]$ for the testing of multiple hypotheses, to reduce the possibility of making a type II error in the interpretation of results. Pearson correlation coefficients are reported for bivariate correlations. Multivariate stepwise regression modelling was performed to achieve the most predictive model utilising the fewest variables. Predictive variables were entered into the model using an alpha of $\mathrm{p}<0.1$ to accommodate the possibility of variables achieving statistical significance once the confounding influence of additional variables was controlled. All analysis was carried out using Minitab (release 16) software.

\section{Results}

Descriptive data of the anthropometric variables assessed are presented in Table 1 . The mean age of the players was 23 years, with the forwards on average 2 years older than the backs. As expected, the forwards had a substantially 
Table 1 Anthropometric data

\begin{tabular}{|c|c|c|c|c|}
\hline & All players & Forwards & Backs & Significance \\
\hline$n$ & 27 & 16 & 11 & $0.317^{*}$ \\
\hline Age (years) & 22.95 (3.94) & $26.00(4.43)$ & $23.70(2.98)$ & 0.164 \\
\hline Height (cm) & $186.21(6.40)$ & $191.00(6.25)$ & $182.50(3.87)$ & $0.002^{\ddagger}$ \\
\hline Weight (kg) & $97.94(10.73)$ & $107.42(5.47)$ & $89.57(6.01)$ & $<0.001^{\ddagger}$ \\
\hline Neck girth $(\mathrm{cm})$ & $41.90(2.65)$ & $43.41(1.96)$ & $39.55(1.50)$ & $<0.001^{\ddagger}$ \\
\hline Chest girth $(\mathrm{cm})$ & $105.62(5.49)$ & $107.72(4.90)$ & $101.70(4.37)$ & $<0.001^{\ddagger}$ \\
\hline \multicolumn{5}{|l|}{ CROM (degrees) } \\
\hline Flexion & $57.92(9.37)$ & $54.38(7.94)$ & $63.20(8.79)$ & $0.019^{\ddagger}$ \\
\hline Extension & $75.48(11.84)$ & 72.06 (12.97) & $79.40(9.05)$ & 0.103 \\
\hline Left side flexion & $48.84(8.48)$ & $47.38(9.26)$ & $48.20(7.08)$ & 0.80 \\
\hline Right side flexion & $45.20(8.29)$ & $43.88(8.08)$ & $47.60(8.10)$ & 0.268 \\
\hline Left rotation & $78.40(13.17)$ & 72.75 (11.70) & $87.20(9.58)$ & $0.002^{\ddagger}$ \\
\hline Right rotation & $81.32(12.94)$ & 76.75 (12.86) & $88.70(8.59)$ & $0.009^{\ddagger}$ \\
\hline
\end{tabular}

All data reported as mean (SD). CROM = cervical range of motion. ${ }^{*}$ Chi square. ${ }^{\ddagger}$ Remains significant at $p=0.05$ adjusting for multiple comparisons.

larger body mass than the back line, with highly significant differences observed in height, weight and measures of neck and chest circumference $(\mathrm{p}<0.002)$. The forwards presented with a notably reduced cervical range of motion compared to the backline, with significant differences of around 10 degrees observed in measures of flexion and rotation (Table 1, Figure 1).

The largest isometric forces were generated in extension, with broadly equal isometric left and right side flexion forces at around 90\% of peak extension values. The forwards generated significantly larger forces than the backline in all test parameters bar isometric flexion (Table 2, Figure 2). Proportional differences between flexion and extension forces were however consistent between player groups, with flexion $27.56 \%$ (SD 12.02\%) reduced compared to extension in the forwards and $27.50 \%(13.85 \%)$ in the backs. Differences between left and right side flexion forces were similar between forwards and backs [7.19\% (7.61\%) and 8.40\% (7.34\%) respectively], as were differences between left and right isometric rotation $[6.19 \%(4.61 \%)$ and $6.50 \%(3.89 \%)$ in the forwards and backs respectively.

\section{Relationship between neck strength and anthropologic variables}

A strong association was observed between isometric cervical extension and neck circumference $(r=0.65)$, though not with chest circumference $(r=0.27)$. Modest association was observed with body weight $(r=0.49)$. Cervical range of motion in flexion and rotation was modestly associated with isometric extension $(r=0.54$ and 0.40 respectively), extension and side flexion were poorly associated (Table 3). Multivariate stepwise regression modelling of global isometric neck strength revealed neck circumference to be the sole predictor of isometric extension, and accounted for around a third of the

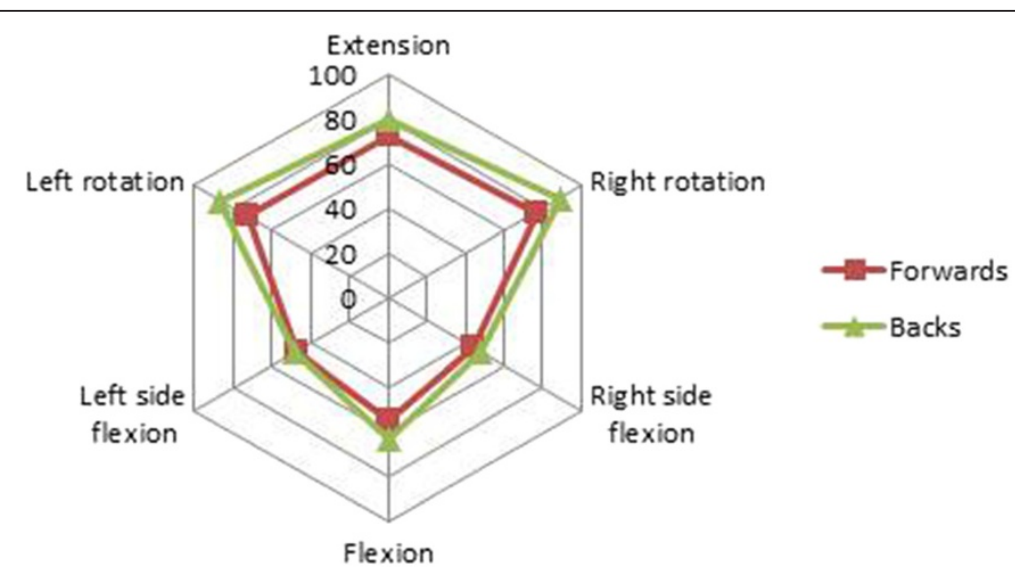

Figure 1 Radar graph demonstrating the pattern of range of motion of the cervical spine of elite rugby players, highlighting differences between forwards and backs. 
Table 2 Cervical isometric neck strength $(\mathbf{k g})$

\begin{tabular}{lllll}
\hline & All players & Forwards & Backs & Significance \\
\hline Flexion & $30.92(5.20)$ & $32.00(5.60)$ & $28.54(3.98)$ & 0.079 \\
Extension & $43.39(6.39)$ & $44.92(7.12)$ & $39.52(5.12)$ & $0.035^{\ddagger}$ \\
Left side flexion & $40.54(6.97)$ & $42.90(7.70)$ & $35.02(4.50)$ & $0.003^{\ddagger}$ \\
Right side flexion & $40.34(7.60)$ & $43.15(7.58)$ & $35.03(4.53)$ & $0.002^{\ddagger}$ \\
Left rotation & $36.09(6.15)$ & $37.56(6.96)$ & $33.08(3.31)$ & $0.006^{\ddagger}$ \\
Right rotation & $36.69(5.38)$ & $38.55(5.52)$ & $33.46(3.04)$ & $0.038^{\ddagger}$ \\
\hline
\end{tabular}

All data reported as mean (SD). ${ }^{\ddagger}$ Remains significant at $p=0.05$ adjusting for multiple comparisons.

variation in cervical isometric extension (adjusted $\mathrm{R}^{2}=$ 30.34) (Figure 3).

\section{Discussion}

This study presents baseline data for the cervical isometric strength and range of motion of UK based professional rugby players. Generally high maximal strength values were observed, with significant differences evident between the forwards and backline players. The forwards were bigger and heavier with correspondingly larger neck circumference. These factors were related to both maximal strength and range of motion; the forwards displaying notably reduced flexion and rotation. Interestingly however in multivariate modelling accounting for confounding variables, only the neck circumference was predictive of maximal isometric strength.

While we would expect strength values of professional athletes to be substantially higher than that of the general population, the values presented here are three times greater than those reported in a healthy Danish population. Jordan et al. (1999) reported values of around $14 \mathrm{Kg}$ for isometric extension for 20-30 year old males [20]. This though was a small cohort of a larger population $(\mathrm{n}=10)$ and these individuals weighed only $80 \mathrm{Kg}$, some $20 \mathrm{Kg}$ lighter than the professional rugby players assessed in this study. More recently, Salo et al. (2006) reported the isometric neck strength of healthy females [25], finding the isometric extension strength of similarly aged women to be around $20 \mathrm{Kg}$, seemingly in proportion to our findings. Again these individuals were markedly smaller than this group at around $167 \mathrm{~cm}$ in height and $62 \mathrm{~kg}$ in weight.

Various equipment and testing protocols have been used in different studies of cervical isometric strength making it difficult to compare findings. A particular problem in comparing to wider literature is the propensity to use isokinetic dynamometers to assess an isometric load. These results are typically presented as torques, though the length of the moment arm employed is rarely defined to allow proper evaluation of the torque values presented. Generally it must be assumed that the moment arm used in these studies relates to the position of the arm of the testing machine relative to its rotational axis. This methodology fails to account however for the length of the individuals' neck, which need also be taken into consideration as this clearly influences the actual torque generated. The only directly comparable data available (using the same testing regime and equipment) is a large crosssectional study of rugby playing Scottish schoolchildren [13]. In this cohort, increasing values were observed between the ages of 12 and 18 with mean isometric extension values ranging between 20 and $30 \mathrm{Kgs}$, again seemingly consistent with this report of professional athletes. The ratio of flexion to extension strength of around $70 \%$ presented here is however consistent across previous reports $[12,19,20,25]$ lending validity to these results.

An intuitive relationship between isometric neck strength and body mass is typically reported, though the strength of this association is poorly defined, perhaps partly due to the difficulty noted in comparing studies and also perhaps that previous studies have assessed various healthy and clinical populations. In these elite rugby players, though

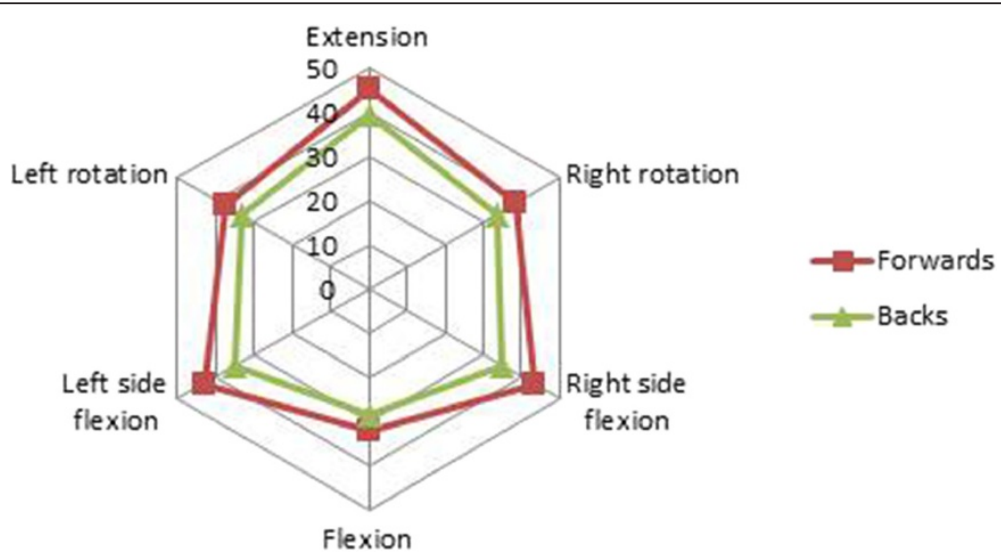

Figure 2 Radar graph demonstrating the pattern of isometric strength of the cervical spine of elite rugby players, highlighting differences between forwards and backs. 
Table 3 Bivariate analysis of predictors of global isometric neck strength

\begin{tabular}{lll}
\hline Predictor variable & $\mathbf{r}$ & Significance \\
\hline Age & 0.27 & 0.224 \\
Weight & 0.49 & 0.034 \\
Height & 0.27 & 0.267 \\
Neck girth & 0.65 & 0.001 \\
Chest girth & 0.26 & 0.205 \\
CROM flexion & -0.54 & 0.005 \\
CROM extension & -0.15 & 0.471 \\
CROM left side flexion & -0.10 & 0.645 \\
CROM right side flexion & -0.13 & 0.545 \\
CROM left rotation & -0.38 & 0.059 \\
CROM right rotation & -0.40 & 0.049 \\
\hline
\end{tabular}

increased player weight and reduced cervical rotation were certainly associated with stronger necks, the only relevant predictor in our modelling was neck circumference. It has been previously shown that neck muscle cross-sectional area is related to muscle strength [26]. Interestingly, in a previous assessment of adolescent rugby players we found that neck circumference was not associated with cervical strength, but that a regression algorithm using player age, weight and grip strength was strongly predictive of isometric extension [13]. In the physically mature rugby players assessed here, a comparatively strong association is observed; highlighting that once fully developed, changes in neck circumference are likely to reflect the muscle volume of the individual. This larger muscle mass is also likely to be associated with the reduced flexion and rotation seen in the forward players.
The primary limitation of this study is the comparatively small sample size and therefore the wider generalisiblity. The number of participants is consistent with most other work in this area and this limitation is mitigated by assessing all available players in a representative elite rugby club. This group is likely to represent a relatively homogeneous population of highly trained individuals reflective of their peers. The values reported here are notably higher than those seen in untrained populations, and in younger players, as would be expected. It must also be noted that this assessment was a single point in time, and we cannot therefore comment as to whether strength and range of motion parameters change over the season. It is reasonable to suggest that enhanced strength and flexibility are developed with training interventions over the season and (as with the wider musculoskeletal system) particular attention should be paid in the pre-season conditioning phase to mitigate injury. Further work is however needed to confirm this suggestion.

\section{Conclusion}

Rehabilitative training programs typically aim to restore the individual to pre injury status. This work provides reference ranges for the strength and range of movement of the cervical spine of current elite level UK based rugby players. In this group, neck strength is most associated with neck circumference, which reflects the underlying cross-sectional muscle area, though the predictive value of this parameter was comparatively low, explaining around a third of the variation in strength. Ultimately, as with any rehabilitative intervention, specific 'pre-injury' parameters of each individual are beneficial to appropriately guide rehabilitation and conditioning programmes.

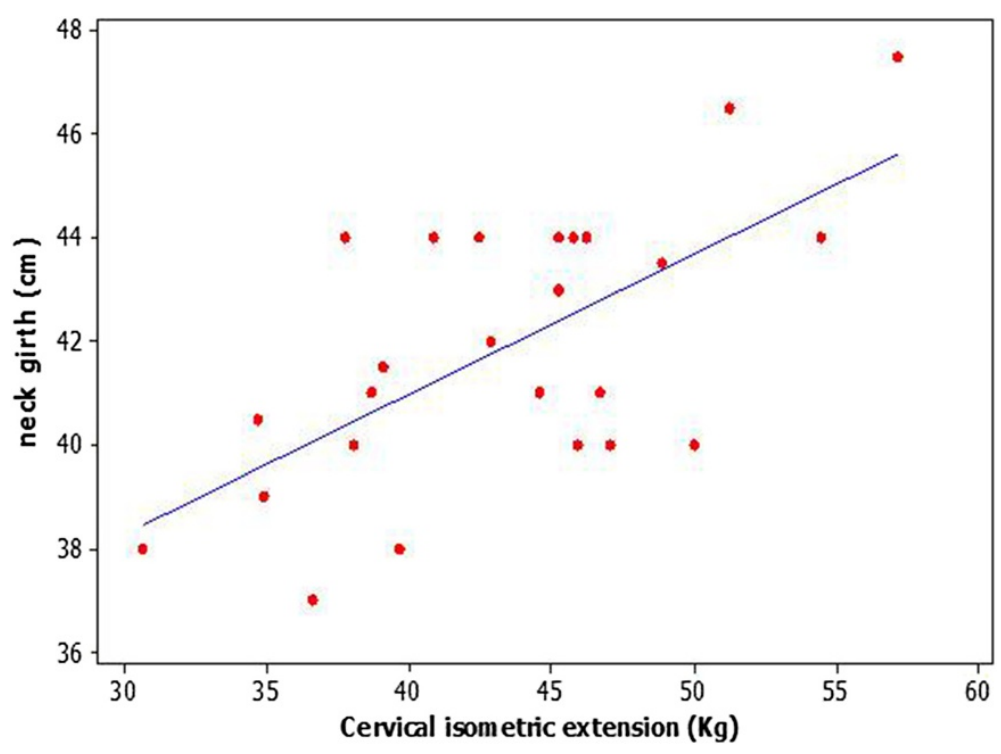

Figure 3 Relationship between global neck strength and neck circumference $\left(R^{2}=30.34\right)$. 


\section{Competing interests}

DG has shares in Gatherer Systems which provided the cervical test equipment for this study. DH declares no competing interests.

\section{Authors' contributions}

Both authors designed the study, DG collected the data, DH analysed the data, $\mathrm{DH}$ drafted the manuscript, both authors revised and approved the final submission.

\section{Acknowledgements}

This work received no specific funding. We wish to thank the medical team and players for their support in conducting this project.

\section{Author details}

'Department of Trauma and Orthopaedics, University of Edinburgh, Chancellor's Building, 49 Little France Crescent, Edinburgh EH164SB, UK.

${ }^{2}$ Gatherer (Physiotherapy Limited), Tudor House, Aylesbury, UK.

Received: 31 January 2014 Accepted: 14 July 2014

Published: 31 July 2014

\section{References}

1. Sedeaud A, Marc A, Schipman J, Tafflet M, Hager JP, Toussaint JF: How they won Rugby World Cup through height, mass and collective experience. Br J Sports Med 2012, 46:580.

2. Murray AD, Murray IR, Robson J: Rugby Union: faster, higher, stronger: keeping an evolving sport safe. Br J Sports Med 2012, 0:1-2. doi:10.1136/bjsports-2012-091844.

3. Duthie $G$, Pyne $D$, Hooper S: Applied physiology and game analysis of rugby union. Sports Med 2003, 33:973-991.

4. Quarrie KL, Hopkins WG: Changes in player characteristics and match activities in Bledisloe Cup rugby union from 1972 to 2004. J Sports SC 2007, 25:895-903.

5. Palmer-Green DS, Stokes KA, Fuller CW, England M, Kemp SP, Trewartha G: Match injuries in English youth academy and schools rugby union: an epidemiological study. Am J Sports Med 2013, 41:749-755.

6. Brooks JHM, Kemp SPT: Injury-prevention priorities according to playing position in professional rugby union players. Br J Sports Med 2011, 45:765-775.

7. Torg J, Ramsey-Emrhein J: Suggested management guidelines for participation in collision activities with congenital, developmental, or post injury lesions involving the cervical spine. Med Sci Sports Exerc 1997, 29(7):256-272

8. Lark SD, McCarthy PW: Cervical range of motion and proprioception in rugby players versus non-rugby players. J Sports Sci 2007, 25:887-894.

9. Pinsault N, Anxionnaz M, Vuillerme N: Cervical joint position sense in rugby players versus non-rugby players. Phys Ther Sport 2010, 11:66-70.

10. Bottini E, Poggi EJ, Luzuriaga F, Secin FP: Incidence and nature of the most common rugby injuries sustained in Argentina (1991-1997). Br J Sports Med 2000, 34:94-97.

11. McIntosh AS, McCrory P, Finch CF, Wolfe R: Head, face and neck injury in youth rugby: incidence and risk factors. Br J Sports Med 2010, 44:188-193.

12. Olivier PE, Du Toit DE: Isokinetic neck strength profile of senior elite rugby union players. J Sci Med Sport 2008, 11:96-105.

13. Hamilton DF, Gatherer D, Jenkins PJ, Maclean JGB, Hutchison JD, Nutton RW, Simpson AHRW: Age-related differences in the neck strength of adolescent rugby players: A cross-sectional cohort study of scottish schoolchildren. Bone Joint Res 2012, 1:152-157.

14. Sarig-Bahat $\mathrm{H}$ : Evidence for exercise therapy in mechanical neck disorders. Man Ther 2003, 8:10-20.

15. Tousignant M, De Bellefeuille L, O'Donoughue S, Grahovac S: Criterion validity of the cervical range of motion (CROM) goniometer for cervical flexion and extension. Spine (Phila Pa 1976) 2000, 25:324-330.

16. Tousignant M, Duclos E, Laflèche $S$, Mayer A, Tousignant-Laflamme $Y$, Brosseau L, O'Sullivan JP: Validity study for the cervical range of motion device used for lateral flexion in patients with neck pain. Spine (Phila Pa 1976) 2002, 27:812-817.

17. Audette I, Dumas JP, Côté JN, De Serres SJ: Validity and between-day reliability of the cervical range of motion (CROM) device. J Orthop Sports Phys Ther 2010, 40:318-323.
18. Youdas JW, Garrett TR, Suman VJ, Bogard CL, Hallman HO, Carey JR: Normal range of motion of the cervical spine: an initial goniometric study. Phys Ther 1992, 72:770-780.

19. Garge's GL, Medina D, Milutinovic L, Garavote P, Guerado E: Normative database of isometric cervical strength in a healthy population. Med Sci Sports Exerc 2002, 33:464-470.

20. Jordan A, Mehlsen J, Bülow PM, Ostergaard K, Danneskiold-Samsoe B: Maximal isometric strength of the cervical musculature in 100 healthy volunteers. Spine 1999, 24:1343-1348.

21. Chiu TT, Sing KL: Evaluation of cervical range of motion and isometric neck muscle strength: reliability and validity. Clin Rehabil 2002, 16:851-858.

22. Peek $\mathrm{K}$, Gatherer D: The rehabilitation of a professional rugby union player following a C7/T1 posterior microdiscectomy. Phys Ther Sport 2005, 6:195-200.

23. Benjamini Y, Hochberg Y: Controlling the false discovery rate: a practical and powerful approach to multiple testing. J R Statist Soc B 1995, 57:289-300.

24. Storey JD: "The positive false discovery rate: $A$ Bayesian interpretation and the q-value". Ann Stat 2003, 31:2013-2035.

25. Salo PK, Ylinen JJ, Mälkiä EA, Kautiainen H, Häkkinen AH: Isometric strength of the cervical flexor, extensor, and rotator muscles in 220 healthy females aged 20 to 59 years. J Orthop Sports Phys Ther 2006, 36:495-502.

26. Mayoux-Benhamou MA, Wybier M, Revel M: Strength and cross-sectional area of the dorsal neck muscles. Ergonomics 1989, 32:513-518.

doi:10.1186/2052-1847-6-32

Cite this article as: Hamilton and Gatherer: Cervical isometric strength and range of motion of elite rugby union players: a cohort study. BMC Sports Science, Medicine, and Rehabilitation 2014 6:32.

\section{Submit your next manuscript to BioMed Central and take full advantage of:}

- Convenient online submission

- Thorough peer review

- No space constraints or color figure charges

- Immediate publication on acceptance

- Inclusion in PubMed, CAS, Scopus and Google Scholar

- Research which is freely available for redistribution

Submit your manuscript at www.biomedcentral.com/submit
Ciomed Central 